\title{
First report of a new corneal pathogen: Phaeoacremonium parasiticum
}

\author{
Horace Massa ${ }^{1}$ (D) Arnaud Riat $^{2} \cdot$ Georgios D. Panos ${ }^{3}$
}

Received: 5 May 2020 / Accepted: 2 July 2020 / Published online: 12 July 2020

(C) The Author(s) 2020

\begin{abstract}
Keratitis is a public health issue in developing countries and a potentially sight-threatening condition. Collagen fibrils in the corneal stroma are parallels to each other. Fundamental substance maintains the same space between collagen fibrils. That is how corneal transparency can be achieved. Any damage which can modify this structure will lead to corneal opacity and loss of vision. Fungal keratitis might appear in up to one-third of cases. Nevertheless, fungal keratitis remains poorly described and understood. Herein, we present the first ever reported case of corneal infection due to Phaeoacremonium parasiticum in a young patient. We describe the clinical and microbial characteristics, and we also discuss the use of confocal microscopy in early diagnosis of this infection.
\end{abstract}

Keywords Keratitis $\cdot$ Eye $\cdot$ Fungus $\cdot$ Microbiology $\cdot$ Confocal in vivo imaging $\cdot$ Phaeoacremonium

\section{Introduction}

Corneal-related disorders are the fifth cause of blindness worldwide [1]. Any damage which will affect the corneal anatomy will lead to corneal opacity [2]. Collagen fibrils in the corneal stroma are parallels to each other. Fundamental substance maintains the same space between each collagen fibril, that is how corneal transparency can be achieved [3].

Infectious keratitis will induce corneal scarring $[4,5]$. Therefore, accurate and prompt diagnosis is of outmost importance for patient prognosis and quality of life [6].

Bacterial keratitis remains the main cause of infectious keratitis, with a prevalence over $90 \%$ in northern USA, but fungal keratitis might appear in up to one-third of cases in more southern locations of the country [7]. Moreover, fungal keratitis has a longer healing time and leads to five times more corneal perforations, which makes it a much more severe condition [8].

Horace Massa

Horace.massa@hcuge.ch

1 Department of Ophthalmology, Geneva University Hospitals, Rue Alcide - Jentzer 22, 1211 Genève 14, CH, Switzerland

2 Service of Laboratory Medicine, Department of Diagnostic, Geneva University Hospitals and Geneva University, Geneva, Switzerland

3 Eye Treatment Centre, Whipps Cross University Hospital, Barts Health NHS Trust, London, UK
Appropriate diagnosis of fungal keratitis and identification of fungal isolates to species is of utmost importance as some fungi might be unresponsive to conventional antifungal treatment [9].

We, herein, present the first ever reported case of corneal infection due to Phaeoacremonium parasiticum in a young female patient. The patient provided written consent form.

\section{Case description}

A 30-year-old female patient attended our eye casualty complaining of pain in the right eye.

History revealed that she was wearing monthly colored contact lenses for cosmetic purposes over the last 2 weeks. She had no history of trauma nor immunosuppression. Five days prior to her presentation, she had a consultation with her ophthalmologist in Brazil and was diagnosed with keratitis. She was discharged from the doctor's clinic with gentamycin drops 5 times a day which improved slightly her symptoms.

Upon presentation at Geneva University Hospital, corneal sensitivity was preserved. Visual acuity was $5 / 10$ Snellen unaided and could only be improved to 10/10 with pinhole.

At slit lamp examination, the cornea was clear except the center where small sub epithelial infiltrates were clearly visible with some fluorescein staining, but surprisingly the conjunctiva was calm. 
Instead of gentamycin, the patient received a prescription of moxifloxacin drops 6 times a day.

Two days later, the patient came back with a deterioration of her condition. She was experiencing pain, decreased vision and photophobia (Fig. 1).

The conjunctiva was inflamed, the cornea was diffusely oedematous, with sub epithelial infiltrates staining with fluorescein and signs of inflammations (cells $0.5+$, small retro descemetic precipitates $1+$ ) were visible in the anterior chamber. Neither perineuritis nor deep stromal abscess was present.

Corneal scrapes were collected, the patient was admitted and chlorhexidine combined with desomedine local drops given hourly was initiated.

At day 1 after hospitalization, corneal confocal microscopy revealed the presence of filaments in the anterior stroma with no evidence of cysts (Fig. 2) and the direct microscopy examination (Fungi Fluor kit) in the laboratory confirmed the diagnosis of a filamentous fungus. The treatment was switched to liposomal amphotericin B and voriconazole drops hourly with desomedine every $2 \mathrm{~h}$ until the amoebic PCR came back negative after 4 days.

The patient was seen daily with daily corneal scrapes for 4 days to diminish the load of filament and to allow a better penetration of antifungal drops.

After 6 days of incubation at $30^{\circ} \mathrm{C}$, the growth of a filamentous fungus was noticeable on Sabouraud agar. The cotton blue microscopic analysis revealed germ associated with the fungus Acremonium sp. However, neither the microscopic nor the mass spectrometry (MALDI-TOF, Biotyper 4.1 Bruker Daltonic, Bremen, Germany) analysis could identify the pathogen. The filamentous fungus responsible for the keratitis was identified by internal transcribed spacer (ITS) sequencing as Phaeoacremonium parasiticum with $99.6 \%$ similarity (GenBank: AB190405.1) (Fig. 3-image filament in culture). Antifungal susceptibility testing was performed using a standardized broth microdilution method (Sensititre ${ }^{\mathrm{TM}}$

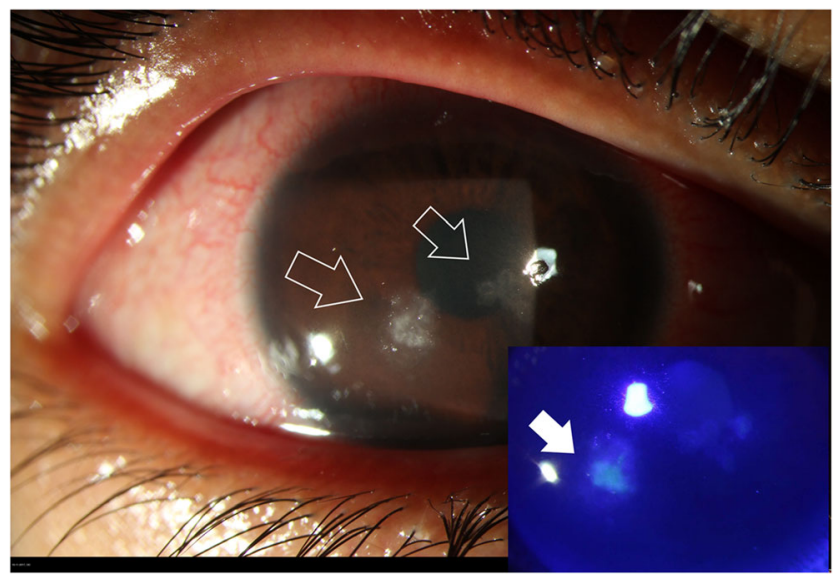

Fig. 1 Slit lamp images of the right eye: white arrows pointing the fungal keratitis with one satellite lesion. Bottom right, the same image with fluorescein test revealing epithelium damage and the stromal impregnation of the yellowish dye (full white arrow)

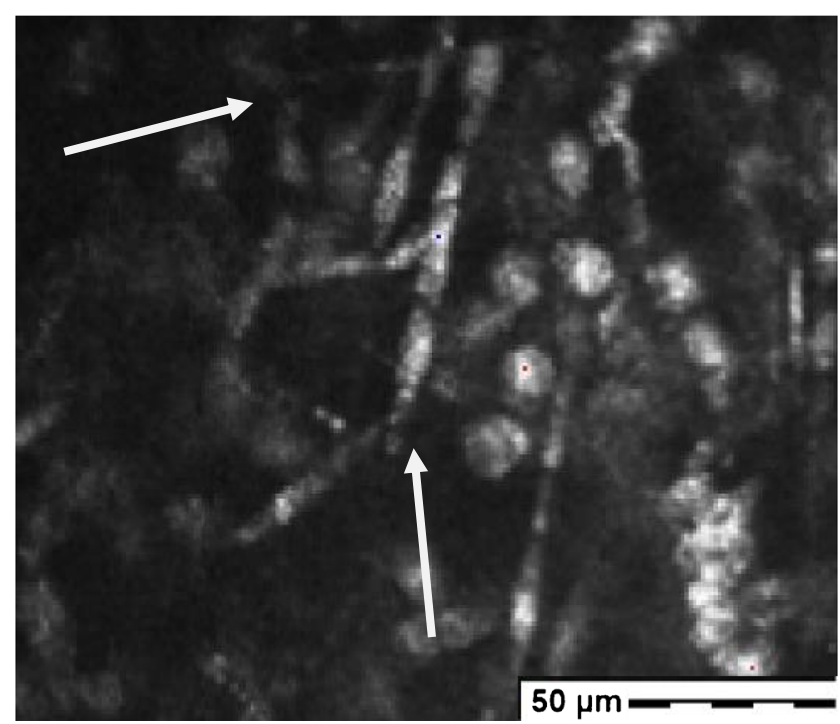

Fig. 2 Image of the corneal lesion showing hyper reflective round structure corresponding to inflammatory cells and the presence of highly reflective fungal hyphae (white arrows) at 70 microns stromal depth (picture acquired with the Heidelberg Retina Tomograph II Rostock Cornea Module)

YeastOne $^{\mathrm{TM}}$ ). The Minimal Inhibitory Concentrations (MIC) of amphotericin B, fluconazole, itraconazole, posaconazole and voriconazole are respectively $1 \mu \mathrm{g} / \mathrm{ml}, 64 \mu \mathrm{g} / \mathrm{ml}, 16$ $\mu \mathrm{g} / \mathrm{ml}, 0.12 \mu \mathrm{g} / \mathrm{ml}$ and $0.12 \mu \mathrm{g} / \mathrm{ml}$.

Other laboratory tests including HIV serology, bacterial culture, herpes PCR and amibian PCR were negative.

As clinical evolution was favorable, no systemic treatment was considered. After 10 days, the patient was discharged with topical treatment of voriconazole and liposomal amphotericin B (1 drop 8 times a day for both). At day 1 after discharge, the vision was $4 / 10$ (Snellen units) unaided and 6/10 with pinhole. The cornea had still 2 paracentral infiltrates with no more

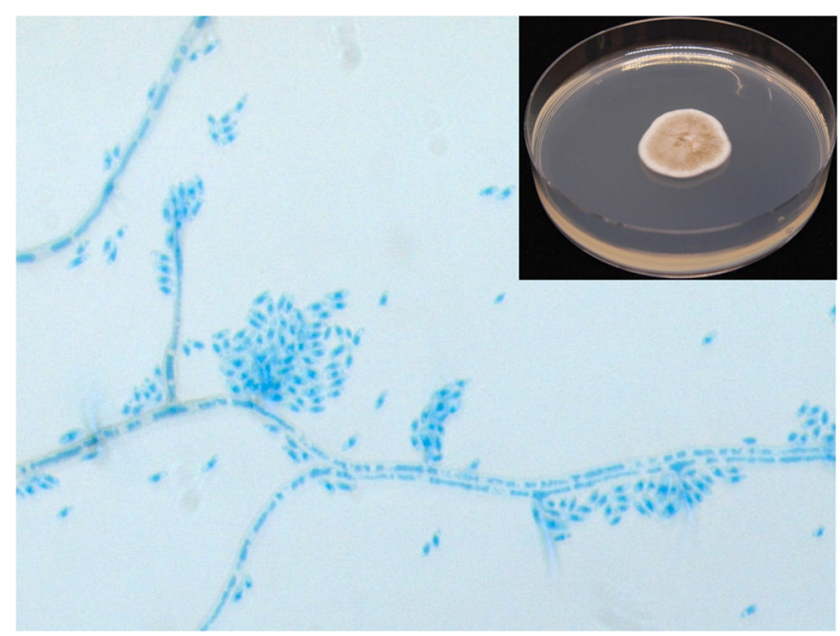

Fig. 3 Main picture: lactophenol cotton blue stain reveals phialides bearing apical clusters of cylindrical to sausage-shaped hyaline conidia. Top left: the colonies have a moderate growth in Sabouraud agar at $30^{\circ} \mathrm{C}$; the appearance of the colonies is velvety, white-grey to brown with radial furrows 
surrounding inflammatory stroma and no more staining. Anterior chamber was also quiet. At day 5 after discharge, and 15 days of topical antifungal treatment, the treatment was discontinued with no recurrence of the infection after 2 weeks.

At 1-year follow-up visit, visual acuity was 1.0 unaided (Fig. 4).

\section{Discussion}

As abovementioned, it is important that the clinician is aware of the new pathogens. Phaeoacremonium parasiticum is a known pathogen in the agricultural domain, and especially in grapevine culture [10].

The first human case reported was in 1974 [11]. Since then, an increasing amount of human infections have been reported, especially in immunocompromised patients as they are opportunistic pathogens [12]. In ophthalmology, only one case has been reported to our knowledge, as a late-onset of posttraumatic endophthalmitis due to a phaeoacremonium [13].

Since almost all germs are unable to penetrate a healthy corneal epithelium, the history of ocular trauma should always be investigated.

In our case, the patient had two major risk factors: the patient was a contact lens wearer coming from a tropical country. As mentioned above, fungal keratitides are more frequent in tropical countries. Also fungal aetiology differs since filamentous keratitis is more frequent in tropical region, whereas in temperate countries, yeast represents the most common cause of fungal corneal infections [14]. Another important risk factor is the cosmetic contact lenses, as they have an almost 2 times increased risk of developing a keratitis compared with refractive contact lenses [15].
Clinical differentiation between a bacterial or a fungal keratitis is not obvious even for corneal specialists [16], but the failure of the initial antibiotic treatment and the progression of the lesion in the stromal cornea should make the clinician consider fungal keratitis as the differential diagnosis. In this case, two characteristics of the filament infection made the differential diagnosis difficult.

1) The slow growth of the Phaeoacremonium parasiticum both in culture and in the patient's cornea is atypical for a fungus and it is a characteristic clinician should bear in mind before excluding a fungal keratitis.

2) Another characteristic is the sub epithelial spread of the disease, as on grapevine leaf, visible on OCT corneal imaging, with only little destruction of the stroma and circumscribed inflammatory reaction, whereas two-thirds of fungal keratitis usually overcome $33 \%$ depth of the stroma [17].

Confocal in vivo imaging showing filamentous infiltrate in the anterior corneal stroma helped us to consider a differential diagnosis. It is a very useful tool and easily accessible but it highly depends on the observer experience [18]. Moreover, Phaeoacremonium parasiticum's hyphae are thinner than the classical Aspergillus hyphae making the diagnosis even more difficult, but, luckily, we had a positive direct examination and culture with the MALDi ToF.

MALDI ToF revolutionized clinical microbiology in the last 10 years and has proven its identification power not only with bacteria but also with fungi. A correct identification depends mostly on the database, which need to be as robust as diverse. The diversity is the Achilles heel of the method particularly with fungi and their vast kingdom. The latest version of the Bruker database does not contain any spectra of Phaeoacremonium parasiticum. In order to thwart the lack of spectra, some
Fig. 4 Slit lamp image of the cornea with a healthy epithelium and a sub epithelial scare (white arrow) 1 year after discharging the patient. Note that the patient is still wearing cosmetic contact lenses

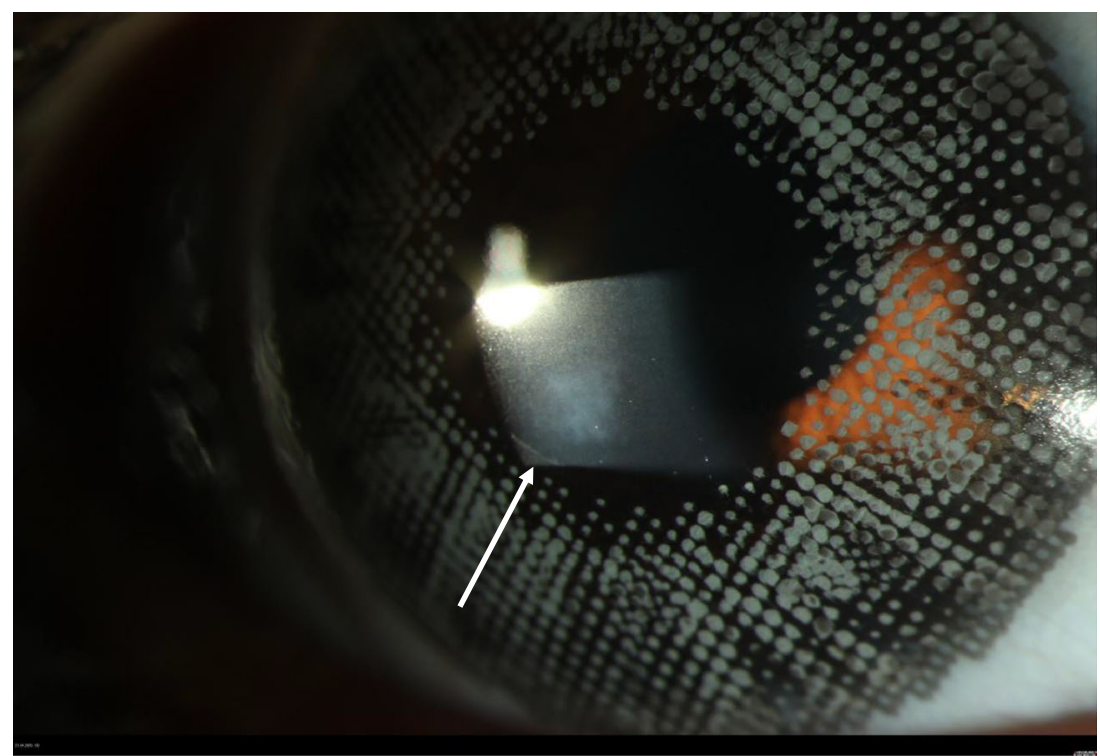


laboratories have developed their own database. The real improvement came from the public database proposed by Pr. Piarroux which is constituted with a large diversity of fungi including Phaeaocremonium parasiticum [19]. At the time of that clinical case, we had to do an ITS sequencing to be able to identify the pathogen. ITS sequencing allows a correct fungal identification in most of the fungi encounter in clinic; however, in comparison with MALDI ToF, it is expensive, time consuming and a highly specialized technician is needed. Thus, the use of a MALDI ToF public database remains a valuable option to identify uncommon clinical fungi.

The in vitro susceptibility profile of Phaeoacremonium parasiticum has been proposed by Badali et al. [20] and shows a similar pattern with Phaeoacremonium parasiticum isolated from the cornea of our patient. Phaeoacremonium parasiticum showed a low MIC for amphotericin B, voriconazole and posaconazole. Fluconazole and itraconazole are in contrary less active. The paucity of data concerning that fungus does not allow any implementation of breakpoint. However, clinical guidelines are proposed by the European Society of Clinical Microbiology and Infectious Disease and European Confederation of Medical Mycology [21].

Clinician and biologists should be aware of this new corneal pathogen-causing keratitis. Unusual evolution should lead to further culture investigations, confocal corneal imaging and prompt instauration of antifungal therapy to achieve the best prognosis.

Funding Information Open access funding provided by University of Geneva.

\section{Compliance with ethical standards}

The authors declare that an informed consent for publishing the images has been gathered.

Conflict of interest The authors declare that they have no conflict of interest.

Open Access This article is licensed under a Creative Commons Attribution 4.0 International License, which permits use, sharing, adaptation, distribution and reproduction in any medium or format, as long as you give appropriate credit to the original author(s) and the source, provide a link to the Creative Commons licence, and indicate if changes were made. The images or other third party material in this article are included in the article's Creative Commons licence, unless indicated otherwise in a credit line to the material. If material is not included in the article's Creative Commons licence and your intended use is not permitted by statutory regulation or exceeds the permitted use, you will need to obtain permission directly from the copyright holder. To view a copy of this licence, visit http://creativecommons.org/licenses/by/4.0/.

\section{References}

1. Bourne RRA, Jonas JB, Bron AM et al (2018) Prevalence and causes of vision loss in high-income countries and in Eastern and Central Europe in 2015: magnitude, temporal trends and projections. Br J Ophthalmol 102:575-585
2. Hassell JR, Birk DE (2010) The molecular basis of corneal transparency. Exp Eye Res 91:326-335

3. Chen S, Mienaltowski MJ, Birk DE (2015) Regulation of corneal stroma extracellular matrix assembly. Exp Eye Res 133:69-80

4. AlMahmoud T, Elhanan M, Elshamsy MH, Alshamsi HN, AbuZidan FM (2019) Management of infective corneal ulcers in a highincome developing country. Medicine (Baltimore) 98:e18243

5. Menda SA, Das M, Panigrahi A et al (2019) Association of postfungal keratitis corneal scar features with visual acuity. JAMA Ophthalmol 138(2):113-118

6. Ung L, Wang Y, Vangel M et al (2019) Validation of a comprehensive clinical algorithm for the assessment and treatment of microbial keratitis. Am J Ophthalmol 214:97-109

7. Estopinal CB, Ewald MD (2016) Geographic disparities in the etiology of bacterial and fungal keratitis in the United States of America. Semin Ophthalmol 31:345-352

8. Prajna NV, Srinivasan M, Lalitha P et al (2013) Differences in clinical outcomes in keratitis due to fungus and bacteria. JAMA Ophthalmol 131:1088-1089

9. Garg P, Roy A, Roy S (2016) Update on fungal keratitis. Curr Opin Ophthalmol 27:333-339

10. Raimondo ML, Lops F, Carlucci A (2014) Phaeoacremonium italicum sp. nov., associated with esca of grapevine in southern Italy. Mycologia 106:1119-1126

11. Ajello L, Georg LK, Steigbigel RT, Wang CJ (1974) A case of phaeohyphomycosis caused by a new species of Phialophora. Mycologia. 66:490-498

12. Mostert L, Groenewald JZ, Summerbell RC et al (2005) Species of Phaeoacremonium associated with infections in humans and environmental reservoirs in infected woody plants. J Clin Microbiol 43: $1752-1767$

13. Huynh TK, Lee LR, Ellis D (2007) Late-onset post-traumatic Phaeoacremonium parasiticum endophthalmitis. Clin Exp Ophthalmol 35:366-368

14. Bharathi MJ, Ramakrishnan R, Meenakshi R, Padmavathy S, Shivakumar C, Srinivasan M (2007) Microbial keratitis in South India: influence of risk factors, climate, and geographical variation. Ophthalmic Epidemiol 14:61-69

15. Sauer A, Meyer N, Bourcier T, French Study Group for Contact Lens-Related Microbial K (2016) Risk factors for contact lensrelated microbial keratitis: a case-control multicenter study. Eye Contact Lens 42:158-162

16. Dalmon C, Porco TC, Lietman TM et al (2012) The clinical differentiation of bacterial and fungal keratitis: a photographic survey. Invest Ophthalmol Vis Sci 53:1787-1791

17. Dan J, Zhou Q, Zhai H et al (2018) Clinical analysis of fungal keratitis in patients with and without diabetes. PLoS One 13:e0196741

18. Sabour S, Ghassemi F (2017) Sensitivity and specificity of laserscanning in vivo confocal microscopy for filamentous fungal keratitis: role of observer experience. Am J Ophthalmol 182:201-202

19. Gautier M, Ranque S, Normand AC et al (2014) Matrix-assisted laser desorption ionization time-of-flight mass spectrometry: revolutionizing clinical laboratory diagnosis of mould infections. Clin Microbiol Infect 20:1366-1371

20. Badali H, Khodavaisy S, Fakhim H, de Hoog GS, Meis JF, Chowdhary A (2015) In vitro susceptibility profiles of eight antifungal drugs against clinical and environmental strains of Phaeoacremonium. Antimicrob Agents Chemother 59:7818-7822

21. Chowdhary A, Meis JF, Guarro J et al (2014) ESCMID and ECMM joint clinical guidelines for the diagnosis and management of systemic phaeohyphomycosis: diseases caused by black fungi. Clin Microbiol Infect 20(Suppl 3):47-75

Publisher's note Springer Nature remains neutral with regard to jurisdictional claims in published maps and institutional affiliations. 